\title{
A PROBLEM ASSOCIATED WITH A PARTICULAR MARKOV CHAIN
}

\author{
by SAMUEL D. SILVEY
}

(Received 7th June, 1954)

1. Introduction. The problem discussed in this paper is a rather specialised problem associated with a particular random walk with reflecting barriers. Such processes are discussed in particular by Feller (2). It happens that probabilities at any time associated with the system here discussed are relatively easy to determine and it is this fact which makes the given solution possible. This problem arose in certain investigations concerned with computing machines carried out by Mr. A. E. Roy of the Astronomy Department in this University and I wish to thank Mr. Roy for bringing it to my notice.

The system concerned has $2 r+1$ states which will be denoted by $-r,-(r-1), \ldots,(r-1)$, $r$. The transition probabilities are stationary and the transition matrix is the $(2 r+1) \times(2 r+1)$ matrix $P$, where

$$
P=\frac{1}{4}\left[\begin{array}{cccccc}
3 & 1 & 0 & 0 & \ldots & 0 \\
1 & 2 & 1 & 0 & \ldots & 0 \\
0 & 1 & 2 & 1 & \ldots & 0 \\
. & & & & & . \\
. & & & & . \\
. & & & & & . \\
0 & 0 & \ldots \ldots \ldots .1 & 2 & 1 \\
0 & 0 \ldots \ldots \ldots \ldots . . & 1 & 3
\end{array}\right]
$$

The probability associated with the $i$ th state after $t$ intervals of time is denoted by $p_{i t}$, $t=0,1,2, \ldots$, and $\mathbf{p}_{t}$ denotes the column vector with components $p_{i t}, i=r,(r-1), \ldots,-r$.

Thus

$$
\mathbf{p}_{t}=\mathbf{P}^{t} \mathbf{p}_{0}
$$

We define $S_{t}=\sum_{i=-r}^{r} i p_{i t}$. If we apply the general theory of Markov chains (1), it is easy to show that, whatever $\mathrm{p}_{0}$ may be, $S_{t \rightarrow 0}$ as $t \rightarrow \infty$. The problem with which we are concerned is to find $t$ such that $S_{t}=\frac{1}{2} S_{0}$, for the set of initial probabilities $p_{10}=1$, and $p_{i 0}=0, i \neq 1$. This value of $t$ will be called the mean half life of the system.

2. Determination of $\mathrm{P}^{t}$. We will write $n=2 r+1$, and $\theta=\frac{\pi}{2 n}$. Then the latent roots of the matrix $\mathbf{P}$ can be shown to be $\cos ^{2}(j-1) \theta, j=1,2, \ldots, n$, and the normalised latent vector corresponding to the root $\cos ^{2}(j-1) \theta$ is $a_{j}$, say, where the $i$ th component $a_{i j}$ of $a_{i}$ may be written as

$$
a_{i j}=d_{1 j}\left(\frac{2}{n}\right)^{t} \cos \{(2 i-1)(j-1) \theta\}, \quad i=1,2, \ldots, n
$$

$d_{i j}$ taking the value 1 when $i \neq j$, and the value $\frac{1}{\sqrt{ } 2}$ when $i=j$. 
If $\mathbf{A}$ denotes the matrix $\left(a_{i j}\right)$, and $\mathbf{D}$ denotes the diagonal matrix whose $(j, j)$ th element is $\cos ^{2}(j-1) \theta, j=1,2, \ldots, n$, then $\mathbf{P}$ can be expressed in the form $\mathbf{P}=\mathbf{A D A} \mathbf{A}^{\prime}$, where $\mathbf{A}^{\prime}$ denotes the transpose of $\mathbf{A}$. From this we have

$$
\mathbf{P}^{t}=\mathbf{A D}^{t} \mathbf{A}^{\prime}
$$

Hence if $p_{i l}^{(t)}$ denotes the $(i, l)$ th element of $\mathbf{P}^{t}$,

$$
p_{i l}^{(t)}=\frac{2}{n} \sum_{k=1}^{n}\left[d_{1 k}^{2} \cos \{(2 i-1)(k-1) \theta\} \cos ^{2 t}(k-1) \theta \cos \{(2 l-1)(k-1) \theta\}\right] .
$$

Now

$$
2^{2 t} \cos ^{2 t} \phi=2 \sum_{s=0}^{t} d_{s t}^{2}\left(\begin{array}{c}
2 t \\
s
\end{array}\right) \cos 2(t-s) \phi
$$

and so

$$
\begin{aligned}
n 2^{2 t} p_{i l}^{(t)}= & \sum_{k=1}^{n} d_{1 k}^{2}\{\cos [2(i+l-1)(k-1) \theta]+\cos [2(i-l)(k-1) \theta]\} \\
& \left\{2 \sum_{s=0}^{t} d_{s t}^{2}\left(\begin{array}{c}
2 t \\
s
\end{array}\right) \cos 2(t-s)(k-1) \theta\right\} . \\
= & \sum_{s=0}^{t} d_{s t}^{2}\left(\begin{array}{c}
2 t \\
s
\end{array}\right) \sum_{k=1}^{n} d_{1 k}^{2}\left[\cos (k-1) \theta_{1}+\cos (k-1) \theta_{2}+\cos (k-1) \theta_{3}+\cos (k-1) \theta_{4}\right],
\end{aligned}
$$

where

$$
\begin{aligned}
& \theta_{1}=2(i+l-1+t-s) \theta, \\
& \theta_{2}=2(i+l-1-t+s) \theta, \\
& \theta_{3}=2(i-l+t-s) \theta, \\
& \theta_{4}=2(i-l-t+s) \theta .
\end{aligned}
$$

Now $\sum_{k=1}^{n} \cos (k-1) \theta_{1}=\frac{1}{2} \operatorname{cosec} \frac{\theta_{1}}{2}\left[\sin \frac{\theta_{1}}{2}+\sin \left(n \theta_{1}-\frac{\theta_{1}}{2}\right)\right]$, provided $i+l-1+t-s \neq 2 \alpha n$, where $\alpha$ is an integer. Since $n \theta_{1}=(i+l-1+t-s) \pi, \sum_{k=1}^{n} \cos (k-1) \theta_{1}=0$ if $(i+l-1+t-s)$ is even but not of the form $2 \alpha n$, and $\sum_{k=1}^{n} \cos (k-1) \theta_{1}=1$ if $(i+l-1+t-s)$ is odd. And there are similar results for $\sum_{k=1}^{n} \cos (k-1) \theta_{i}, i=2,3,4$. Also if $(i+l-1+t-s)$ is of the form $2 \alpha n$, then $\sum_{k=1}^{n} \cos (k-1) \theta_{1}=n$, and similarly for $\sum_{k=1}^{n} \cos (k-1) \theta_{i}, i=2,3,4$.

Combining these results and using the fact that, for a given value of $s, i+l-1+t-s$ and $i+l-1-t+s$ are both $\left\{\begin{array}{c}\text { even } \\ \text { odd }\end{array}\right\}$ when $i-l+t-s$ and $i-l-t+s$ are $\left\{\begin{array}{c}\text { odd } \\ \text { even }\end{array}\right\}$, we find that $p_{i l}^{(t)}$ can be expressed in the form

$$
p_{i l}^{(t)}=\frac{1}{2^{2 t}} \sum\left(\begin{array}{c}
2 t \\
s
\end{array}\right)
$$

where summation extends over those values of $s$ in the range $0 \leqslant s \leqslant t$ for which

(i) $i+l-1+t-s$ is of the form $2 \alpha n$,

(ii) $i+l-1-t+s$ is of the form $2 \alpha n$,

(iii) $i-l+t-s \quad$ is of the form $2 \alpha n$,

(iv) $i-l-t+s$ is of the form $2 \alpha n$, 
where $\alpha$ is an integer. This can be written in the more convenient form

$$
2^{2 t} p_{i l}^{(t)}=\sum_{1}\left(\begin{array}{c}
2 t \\
i+l+t-1-2 \alpha n
\end{array}\right)+\sum_{2}\left(\begin{array}{c}
2 t \\
i-l+t+2 \alpha n
\end{array}\right),
$$

where in each case summation extends over integral values of $\alpha$, and for $\sum_{1}$,

while, for $\sum_{2}$,

$$
\frac{i+l-t-1}{2 n} \leqslant \alpha \leqslant \frac{i+l+t-1}{2 n},
$$

$$
\frac{-i+l-t}{2 n} \leqslant \alpha \leqslant \frac{-i+l+t}{2 n}
$$

3. Determination of $S_{t}$. We turn now to a discussion of the mean half life for the given set of initial probabilities. For this set $S_{0}=1$, and $\mathbf{p}_{t}$ is the $r$ th column of the matrix $\mathbf{P}^{t}$. Hence

$$
S_{t}=\sum_{i=-r}^{r} i p_{i t}=\sum_{j=1}^{r}(r-j+1)\left(p_{j r}^{(t)}-p_{(2 r+2-j) r}^{(t)}\right)
$$

If $r$ is small the mean half life may be obtained directly by calculation. Here we discuss an approximation to the mean half life for large values of $r$.

In order to avoid the introduction of odd terms due to the presence of $l \pm i$ in the limits of summation of the sums $\sum_{1}$ and $\sum_{2}$ of (2.1) we discuss initially the value of $S_{t}$ when $t$ is of the form $2 A n$ where $A$ is an integer. In this case the limits of summation for $\sum_{1}$ are $-A<\alpha \leqslant A$, for all $i$ and $l$, and for $\sum_{2},-A<\alpha \leqslant A$ if $i<l,-A \leqslant \alpha<A$ if $i>l$, and $-A \leqslant \alpha \leqslant A$ if $i=l$.

Hence for $t=2 A n, S_{t}=T_{1}+T_{2}$, where

$$
\begin{aligned}
2^{2} t T_{1} & =\sum_{j=1}^{r}(r-j+1) \sum_{\alpha=-A+1}^{A}\left\{\left(\begin{array}{c}
2 t \\
j+r+t-1-2 \alpha n
\end{array}\right)-\left(\begin{array}{c}
2 t \\
2 r+2-j+r+t-1-2 \alpha n
\end{array}\right)\right\}, \\
2^{2} t T_{2} & =\sum_{j=1}^{r}(r-j+1)\left\{\sum_{\alpha=-A+1}^{A}\left(\begin{array}{c}
2 t \\
j-r+t+2 \alpha n
\end{array}\right)-\sum_{\alpha=-A}^{A-1}\left(\begin{array}{c}
2 t \\
r+2-j+t+2 \alpha n
\end{array}\right)\right\}+1 .
\end{aligned}
$$

The term $T_{1}$ can be written in the form

$$
\begin{aligned}
& 2^{2 t} T_{1}=\sum_{j=1}^{r} \sum_{\alpha=-A+1}^{A}(r-j+1)\left(\begin{array}{c}
2 t \\
j+r+t-1-2 \alpha n
\end{array}\right) \\
&-\sum_{j=1}^{r} \sum_{\alpha=-A+1}^{A}(r-j+1)\left(\begin{array}{c}
2 t \\
j+r+t+1+2(\alpha-1) n
\end{array}\right) \\
&\left(\begin{array}{c}
2 t \\
k
\end{array}\right)=\left(\begin{array}{c}
2 t \\
2 t-k
\end{array}\right)
\end{aligned}
$$

since

and $n=2 r+1$. Writing $\alpha$ for $-(\alpha-1)$ in the second of the two sums we obtain

$$
T_{1}=\sum_{j=1}^{r} \sum_{\alpha=-A+1}^{A}(r-j+1)\left\{\frac{1}{2^{2 t}}\left(\begin{array}{c}
2 t \\
j+r+t-1-2 \alpha n
\end{array}\right)-\frac{1}{2^{2 t}}\left(\begin{array}{c}
2 t \\
j+r+t+1-2 \alpha n
\end{array}\right)\right\} .
$$

If we apply the normal approximation to the terms of the binomial distribution $\left(\frac{1}{2}+\frac{1}{2}\right)^{2 t}$ we find that, for large $r$,

$$
\begin{aligned}
T_{1} \sim \frac{1}{\sqrt{ }(\pi t)} \sum_{\alpha=-A+1}^{A}\left\{r e^{-\frac{(r-2 \alpha n)^{3}}{t}}\right. & +(r-1) e^{\left.-\frac{(r+1-2 \alpha n)^{2}}{t}\right\}} \\
& -2 \sum_{\alpha=-A+1}^{A}\left\{\phi\left(\frac{2 r-1-2 \alpha n}{\sqrt{ }\left(\frac{1}{2} t\right)}\right)-\phi\left(\frac{r+2-2 \alpha n}{\sqrt{ }\left(\frac{1}{2} t\right)}\right)\right\},
\end{aligned}
$$


where

$$
\phi(\lambda)=\frac{1}{\sqrt{ }(2 \pi)} \int_{-\infty}^{\lambda} e^{-\mathrm{l} x x^{2}} d x
$$

Now

$$
\begin{aligned}
& \sum_{\alpha=-A+1}^{A}\left\{\phi\left(\frac{2 r-1-2 \alpha n}{\sqrt{ }\left(\frac{1}{2} t\right)}\right)-\phi\left(\frac{r+2-2 \alpha n}{\sqrt{ }\left(\frac{1}{2} t\right)}\right)\right\} \\
= & \sum_{\alpha=1}^{A}\left\{\phi\left(-\frac{2 \alpha n-2 r+1}{\sqrt{ }\left(\frac{1}{2} t\right)}\right)-\phi\left(-\frac{2 \alpha n-r-2}{\sqrt{ }\left(\frac{1}{2} t\right)}\right)\right\} \\
+ & \sum_{\alpha=-A+1}^{0}\left\{1-\phi\left(\frac{2 \alpha n-2 r+1}{\sqrt{ }\left(\frac{1}{2} t\right)}\right)-1+\phi\left(\frac{2 \alpha n-r-2}{\sqrt{ }\left(\frac{1}{2} t\right)}\right)\right\}, \\
= & \sum_{\alpha=1}^{A}\left\{\phi\left(-\frac{2 \alpha n-2 r+1}{\sqrt{ }\left(\frac{1}{2} t\right)}\right)-\phi\left(-\frac{2 \alpha n-r-2}{\sqrt{ }\left(\frac{1}{2} t\right)}\right)\right\} \\
+ & \sum_{\alpha=1}^{A}\left\{\phi\left(-\frac{2 \alpha n-3 r}{\sqrt{ }\left(\frac{1}{2} t\right)}\right)-\phi\left(-\frac{2 \alpha n-2 r-3}{\sqrt{ }\left(\frac{1}{2} t\right)}\right)\right\} \\
\sim & \sum_{\alpha=1}^{A}\left\{\phi\left(-\frac{2 \alpha n-3 r}{\sqrt{ }\left(\frac{1}{2} t\right)}\right)-\phi\left(-\frac{2 \alpha n-r-2}{\sqrt{ }\left(\frac{1}{2} t\right)}\right)\right\}
\end{aligned}
$$

since

$$
\phi\left(-\frac{2 \alpha n-2 r-3}{\sqrt{ }\left(\frac{1}{2} t\right)}\right) \sim \phi\left(-\frac{2 \alpha n-2 r+1}{\sqrt{ }\left(\frac{1}{2} t\right)}\right)
$$

for large $r$, and $t$ is of the form $2 A n$, i.e., $2 A(2 r+1)$.

It follows that

$$
T_{1} \sim \frac{2 r}{\sqrt{(\pi t)}} \sum_{\alpha=-A+1}^{A} e^{-\frac{(r-2 \alpha n)^{2}}{t}}-2 \sum_{\alpha=1}^{A}\left\{\phi\left(-\frac{2 \alpha n-3 r}{\sqrt{ }\left(\frac{1}{2} t\right)}\right)-\phi\left(-\frac{2 \alpha n-r-2}{\sqrt{ }\left(\frac{1}{2} t\right)}\right)\right\} .
$$

Similarly it may be shown that

$$
\begin{aligned}
& T_{2} \sim-\frac{2 r}{\sqrt{ }(\pi t)} \sum_{\alpha=-A+1}^{A} e^{-\frac{(r-2 \alpha n)^{2}}{t}}+2\left\{\phi(0)-\phi\left(-\frac{r-1}{\sqrt{ }\left(\frac{1}{2} t\right)}\right)\right\} \\
& +2 \sum_{\alpha=1}^{A-1}\left\{\phi\left(-\frac{2 \alpha n-r+1}{\sqrt{ }\left(\frac{1}{2} t\right)}\right)-\phi\left(-\frac{2 \alpha n+r-1}{\sqrt{ }\left(\frac{1}{2} t\right)}\right)\right\}+2\left\{\phi\left(-\frac{t-r-1}{\sqrt{ }\left(\frac{1}{2} t\right)}\right)-\phi\left(-\frac{t-2}{\sqrt{ }\left(\frac{1}{2} t\right)}\right)\right\} .
\end{aligned}
$$

Since $\phi(0)=\frac{1}{2}$, since $S_{t}=T_{1}+T_{2}$, and since

$$
\phi\left(-\frac{(2 k+1) r+k+2}{\sqrt{ }\left(\frac{1}{2} t\right)}\right)+\phi\left(-\frac{(2 k+1) r+k-1}{\sqrt{ }\left(\frac{1}{2} t\right)}\right) \sim 2 \phi\left(-\frac{(2 k+1)\left(r+\frac{1}{2}\right)}{\sqrt{ }\left(\frac{1}{2} t\right)}\right),
$$

it follows from the above asymptotic expressions for $T_{1}$ and $T_{2}$ that

$$
S_{t} \sim 1-4 \sum_{k=0}^{2 A-1}(-1)^{k} \phi\left(-\frac{(2 k+1)\left(r+\frac{1}{2}\right)}{\sqrt{ }\left(\frac{1}{2} t\right)}\right) .
$$

This asymptotic expression for $S_{t}$ was derived on the assumption that $t$ was of the form $2 A n$. If $t$ is not of this form, say $t=2 A n+c$ where $0<c<2 n$, then in the general expression 2.1 for $p_{i l}^{(l)}$, summation over $\alpha$ may have to be extended over at most one further integral value of $\alpha$. For large values of $r$ the contribution to $p_{i l}^{(t)}$ from this additional value of $\alpha$ is negligible in comparison with the contributions to $p_{i l}^{(t)}$ from all the numerically smaller values of $\alpha$. Hence 3.1 may be taken as a general asymptotic (with respect to $r$ ) form of $S_{t}$ for all $t$. And in this case $A$ is interpreted as the greatest integer $a$ such that $2 a n \leqslant t$. 
4. Determination of mean half life. The problem of determining the mean half life is now simply the problem of solving the equation

$$
\frac{1}{2}=1-4 \sum_{k=0}^{2 A-1}(-1)^{k} \phi\left(-\frac{(2 k+1)\left(r+\frac{1}{2}\right)}{\sqrt{\left(\frac{1}{2} t\right)}}\right) .
$$

To obtain an approximate solution of this equation we take only the first term in the sum in the right hand side and consider the equation

$$
\frac{1}{2}=1-4 \phi\left(-\frac{r+\frac{1}{2}}{\sqrt{ }\left(\frac{1}{2} t\right)}\right) \text {. }
$$

The solution of this equation is $t=2\left[\frac{r+\frac{1}{2}}{\phi^{-1}\left(\frac{1}{8}\right)}\right]^{2}$. Using tables of the normal distribution function we find that $\frac{2}{\left[\phi^{-1}\left(\frac{1}{8}\right)\right]^{2}}=1.5 \ldots$, and so as a first approximation to the solution of the equation 4.1, we may take $t=\frac{3}{2}\left(r+\frac{1}{2}\right)^{2}$. For this value of $t$,

$$
\phi\left(-\frac{(2 k+1)\left(r+\frac{1}{2}\right)}{\sqrt{ }\left(\frac{1}{2} t\right)}\right)=\phi\left(-\frac{2}{\sqrt{ } 3}(2 k+1)\right),
$$

and this is negligible for $k=1,2, \ldots$ Hence $t=\frac{3}{2}\left(r+\frac{1}{2}\right)^{2}$ is a good approximation to the mean half life. Furthermore the same type of argument shows that up to at least this time $S_{t}$ is well approximated by $1-4 \phi\left(-\frac{2 r+1}{\sqrt{(2 t)}}\right)$, for large values of $r$.

Finally to illustrate how rapidly the mean half life settles down to the asymptotic value $\frac{3}{2}\left(r+\frac{1}{2}\right)^{2}$ we compare the values given by this formula with the true values for $r=1,2,3$, obtained by direct calculation. The figures in the table are taken to the nearest integer.

$\begin{array}{ccc}r & \text { True mean half life } & \frac{3}{2}\left(r+\frac{1}{2}\right)^{2} \\ 1 & 2 & 3 \\ 2 & 8 & 9 \\ 3 & 18 & 18\end{array}$

Acknowledgement. I wish to acknowledge the help I received with this problem from my colleague Mr. W. A. Donaldson who provided many useful suggestions during frequent discussions of it.

\section{REFERENCES}

(1) Doob, J. L., Stochastic Processes (New York, 1953), pp. 170-185.

(2) FELLER, W., An introduction to probability theory and its applications, Vol. 1 (New York, 1950), pp. 279-362.

\section{UNIVERSITY OF GLasqow.}

